Research Article

\title{
Improved Efficient Projection Density Function Based on Topology Optimization
}

\author{
Nouman Saeed $\left(\mathbb{D},{ }^{1}\right.$ Kai Long $\left(\mathbb{D},{ }^{1}\right.$ Jamshed Ahmed Ansari $\left(\mathbb{D},{ }^{2}\right.$ Nasif Raza Jaffri $(\mathbb{D})^{3}$ \\ and Usama Abrar iD ${ }^{3}$ \\ ${ }^{1}$ State Key Laboratory for Alternate Electrical Power System with Renewable Energy Sources, \\ North China Electric Power University, Beijing 102206, China \\ ${ }^{2}$ Deparment of Electrical Engineering, Sukkur IBA University, Sukkur 65200, Pakistan \\ ${ }^{3}$ School of Control and Computer Engineering, North China Electric Power University, Beijing 102206, China
}

Correspondence should be addressed to Kai Long; longkai1978@163.com

Received 8 May 2021; Revised 8 June 2021; Accepted 18 September 2021; Published 8 October 2021

Academic Editor: Riaz Ahmad

Copyright $(92021$ Nouman Saeed et al. This is an open access article distributed under the Creative Commons Attribution License, which permits unrestricted use, distribution, and reproduction in any medium, provided the original work is properly cited.

Topology optimization is a powerful tool having capability of generating new solution to engineering design problems, while these designs enhance manufacturability and reduce manufacturing costs in a computational setting. Mesh-independent convergence and other techniques have been widely used as topology optimization technique, but they produce gray transition regions which is not a favorable condition for any material. In this article, a modified topology optimization formulation using a new function has been proposed. The suggested scheme makes use of the Heaviside Projection Method (HPM) to continuum topology optimization. Such technique is helpful to obtain the minimum length scale influence on void and solid phases. Application of this proposed approach is implemented to obtain the minimum compliance for macrostructures. Numerical remarkable examples illustrate the noteworthy value of the proposed approach.

\section{Introduction}

A process in which the optimization of substance results within a known structural space is used for assumed load parameters and limit circumstances to obtain the top figure occurrence of the structure is called topology optimization. An inspiring effort by Bendsoe and Kikuchi [1] first introduced a sensible design tool to obtain required outcomes for structural optimization problems. The technique appears for the most suitable distribution of material inside a layout space through minimization objective function situation to a set of design constraints. After that, numerous researchers make different kinds of efforts to improve the procedure. Deaton and Grandhi [2] introduced the density-centered method to be prominent because of simple modification to finite element codes. This technique explains metamorphosis of the model layout, iterative aspect, and slow transformation in every new upcoming layout that renovates keen on the dense, outdated, and also immoderate-performance shape. Density-based technique for topology optimization [3] have been developed to such an extent that the structures for numerous engineering categories are implemented using advanced computational means, especially in favor of aerospace and automotive to achieve optimal weight, stiffness, strength, and frequency with the help of design objectives. It is known that the density fundamental variables lie in the range of 1 and 0 [4]. In topology, optimization filtering has the most important role in research subject. Filtering operation is used to find out the resulting structure of geometry, although it has many modifications and fluctuations to examine Heaviside projection. In nondensity topology optimization, to modify length scale, we put in the order level set approach as well as phase-field approach. Density technique is used by means of density filer to sort out merely boundary outcomes. Optimized structure solution cannot be obtained because occasionally it is merely a conceptual layout theory. Therefore, in topology optimization production, constraints have an essential role for the 
solution of the optimized structure. Lazarov et al. [5] have already deliberated in detail.

A large number of researchers have played a role to improve computational competence. Just like that, for minimization weight with several constraints, Long Kai et al. [6] improved capable, diagonally quadratic design. Maruyama et al. [7] established a methodology of an external variable to find out the design problem of optimal topology by means of incorporating external variables, where both the external variables and the material distribution are at the same time as optimized. Zhang et al. [8] used the topology optimization method by the means of moving morphable components for a rib-stiffened structure. In moving morphable components' structure, more than one substance can be executed without difficulty, in addition, reducing significant number of structure variables and degree of freedoms in evaluation. Parr [9] used robust optimization to modify design insensitivity and structural parameters. There are numerous kinds of problems, such as geometric hurdles [10], functioning loading problems [11], and substance problems [12]. In the matter of loading problems, Calafiore and Dabbene [13] explained two different techniques aimed at producing nondeterministic structure layout, which are useful to minimize, particularly, structure coupled with mean value objective problems. Zhao and Wang [14] worked at the robust optimization of isotropic substance design along with loading variability problems. Similarly, many other researchers worked at robust topology optimization for improvement and sustainability. Long et al. [15] explained that topological optimizes that the problem could be well posed as a second-order quadratic. There are different kinds of topology optimization expressions, such as evolutionary technique, bidirectional evolutionary technique [16], homogenization technique [17], and solid isotropic material with penalization techniques $[18,19]$, already operating magnificently to solve the different kinds of structural issues and took appreciated notice from different engineering disciplines. These abovementioned popular approaches are utilized to solve a wide range of topological optimization difficulties in explicit and implicit process. To distinguish the optimal structural topology in solid isotropic material with penalization, we identify black and white allocation through a pixel image; similarly, we take out the level set from TDF specified at given structure limitation. Both, the level set technique and solid isotropic material with penalization (SIMP) technique, solve topology optimization difficulties in an implicit way. We sum up the existing complications with regarding to implicit approach as follows. First of all, it is not easy to manipulate the design feature dimensions. The design feature dimensions play the most important role in production structure. Since it is difficult to create a relation among the optimization model and CAD modeling structure in implicit optimization with due different structural geometric representations without explicit geometric information, researchers proposed a unique procedure to obtain the complete length control [20]. Secondly, the implicit method for the 3D topology optimization problem usually involves many numbers of variables which are required as compared to explicit technique.
When topology optimization (TO) persuades multiphysics, in that case, we obtain difficult numerical problem that put us away from a noteworthy convergence by optimization procedures due to strong coupling of optimization and analysis models.

In this article, we suggest a modified robust formulation using another function. The said formulation associates three projections that are keen on a max-min problem. First, it would be helpful to obtain the minimum length scale influence on void and solid phases to produce a robust, optimized structure. In addition, it attains a brief layout correspondingly $0 / 1$ phases to moderate $\beta$ values. The standard Heaviside filtering method is manipulated to make sure the white and black phases cannot obtain length scale control on void and solid phases in topology optimization [21]. The modified Heaviside projection established scheme can afford to manipulate the length scale on the void phase, and on the opposite side, the original Heaviside projection can afford only minimum length scale control on the solid phase [22]. We cannot obtain length scale influence on both phases, solid and void, by establishing the volume-preserving projection scheme [23].

The rest of this paper is ordered as follows. In Section 2, we explain the essential problem setting (such as explained in Sigmund article) [24]. Section 3 defines robust topology optimization for impression of the three optimized design projections for efficient linear evolution density from 0 to 1 , respectively. Section 4 indicates the numerical examples that certify the efficiency of the implement the newly function. We summarize the most important conclusion in Section 5.

\section{Methodology}

2.1. Topology Optimization Formulation. To treasure out the optimal distribution of material which links up the constraints of the objective function within the simplified mathematical description, prior to fixing the optimization problem, our elementary job is to make its mathematical model according to the basic problem demand. Otherwise, the optimized layout could not be precious. The optimization problem, such as one node associated to hinges acting in complaint mechanism layout, is overlooked. The mathematical method aimed at the essential minimum compliance problem may be proposed such as described by Wang et al. [25]:

$$
\begin{gathered}
\min _{\rho}: f(\rho)=I^{T} u, \\
K u=f, \\
\text { s.t. : } f_{v}(\rho)=\frac{\sum_{i \in N_{e}} \rho_{i} v_{i}}{V} \leq V^{*}, \\
\quad 0 \leq \rho_{i} \leq 1, \quad \forall i \in N_{e},
\end{gathered}
$$

where $K$ is the global stiffness matrix derived through finite element discretization, $u$ and $f$ denote the global displacement and force vectors to the system, respectively, $N_{e}$ 
represents the set of finite element indices, $\widetilde{\tilde{\rho}}_{i}$ is the physical density associated by means of every structure element, $v_{i}$ is the volume of an individual cell $i, v$ signifies the volume of the structure sphere of influence, and $V^{*}$ is the prescribed volume fraction which can be interested by material. In order to simplify the matrix $K$, the element stiffness is associated with material stiffness and represented as $K_{i}=E_{i} K_{0}$, where $K_{0}$ represents unit stiffness coupled with $E_{i}$, which represents the stiffness of material utilizing the SIMP approach, where the material interpolation method can be described as

$$
E_{i}=E_{\min }+\rho_{i}^{p}\left(E_{0}-E_{\min }\right),
$$

where $E_{0}$ and $p$ denote the stiffness of the material and penalization factor $(p \leq 3)$ and $E_{\min }$ represents a precise number the stiffness of void material which helps stiffness matrix not to be singular.

2.2. Sensitivity Analysis. Using the adjoint procedure, sensitivity objective function is calculated corresponding to change in the physical structure variables $\widetilde{\widetilde{\rho}}_{i}$ by adjoint sensitivity analysis as follows:

$$
\frac{\partial f}{\partial \widetilde{\rho}}=\lambda^{T} \frac{\mathrm{d} K}{\mathrm{~d} \rho_{i}} u,
$$

where $\lambda$ represents the global adjoint vector obtained from $K \lambda=-I$.

2.3. Filtering. To control the topology optimization problems, we get through the density-based filter method. First of all, Burns and Tortorelli [26] proposed a process to obtained optimal solution for nonlinear elastic design and yielding procedures; later on, Bourdin [27] verified this mathematically. The essential awareness of the density filter can be expressed as the following two terms. First, for discrete elements in given structure domain nominates density variables which may be constantly changed in specific interval between 0 and 1 . Second, compute the weight coefficient of target elements w.r.t. the function as a consequence find-out filter radius. Finally, the system is capable to find out the filtered density with the help of density-based filter function.

With passing time, many reforms of the algorithm have already been put forward in literature. As an alternative, good form explanation of the layout can be acquired by civilizing the finite element mesh; however, optimization process may add some minor details according to the mesh and converge the solution in a different manner. As a result, the length scale in achieved structure depends on mesh size and likewise goes through from the famous checkerboard pathology on behalf of low-order elements.

According to the key point, the physical element density is a weighted average of the neighbouring structure variables, wherein the neighbourhood is described in the direction of manipulating a circle in $2 \mathrm{D}$ or sphere in $3 \mathrm{D}$ along with respect to distinct radius. Consequently, projection models have been planned in such a way that project-filtered structures in specific interval are presented between 0 and 1 , as proposed by Bruns and Tortorelli [26]:

$$
\tilde{\rho}_{i}=\sum_{j \in N_{e, i}} \omega\left(x_{j}\right) v_{j} \rho_{j} \sum_{j \in N_{e, i}} \omega\left(x_{j}\right) v_{j},
$$

where density filter and projection with specified numbers zero/one represent $\tilde{\rho}_{i}$ and $\widetilde{\widetilde{\rho}}_{i} . N_{e, i}$ is usually identified by the elements that enclose centers in a filter for element $i, \omega\left(X_{j}\right)$ denote the weighting function which is assumed as linearly decaying with corresponding increasing distance. Filter radius is $R$ and $X_{i}$ and $X_{j}$ suppress coordinates at center of the design cells $i$ and $j$. In the following, we defined mathematically $\omega\left(X_{j}\right)$ as

$$
\omega\left(x_{j}\right)=R-\left|x_{j}-x_{i}\right| \text {. }
$$

Now, take the derivative of equation (4) w.r.t. the design variables as well as

$$
\frac{\partial \widetilde{\rho}_{i}}{\partial \rho_{j}}=\omega\left(x_{j}\right) v_{j} \sum_{j \in N_{e, i}} \omega\left(x_{j}\right) v_{j} .
$$

The sensitivities of the objective and the projection function of the filtered density $\widetilde{\widetilde{\rho}}_{i}\left(\widetilde{\rho}_{i}\right)$ are modified applying the chain rule:

$$
\frac{\partial f}{\partial \rho_{j}}=\sum_{i \in N_{e, j}}\left(\frac{\partial f}{\partial \rho_{i}} \frac{\partial \rho_{i}}{\partial \widetilde{\rho}_{i}} \frac{\partial \widetilde{\rho}_{i}}{\partial \rho_{j}}\right)
$$

2.4. Threshold Projection. Xu et al. [28] proposed the projection function with respect to Heaviside function which needs to be have required properties and can be written mathematically as

$$
\rho_{i}=\left\{\begin{array}{l}
0 \leq \widetilde{\rho}_{i} \leq \eta \\
\eta \leq \widetilde{\rho}_{i}<1 .
\end{array}\right.
$$

Here, in equation (8), the $\widetilde{\rho}_{i}$ filtered density and $\eta$ parameter values are lying between zero to one.

By introducing beta $(\beta)$ parameter, the physical density can be modified as

$$
\rho_{i}= \begin{cases}\eta\left[e^{-\beta\left(1-\left(\widetilde{\rho}_{i} / \eta\right)\right)}-\left(1-\frac{\tilde{\rho}_{i}}{\eta}\right) e^{-\beta}\right], & 0 \leq \widetilde{\rho}_{i} \leq \eta, \\ (1-\eta)\left[e^{-\left(\beta\left(\tilde{\rho}_{i}-\eta\right) /(1-\eta)\right)}+\frac{\left(\widetilde{\rho}_{i}-\eta\right) e^{-\beta}}{(1-\eta)}\right]+\eta, & \eta \leq \tilde{\rho}_{i}<1 .\end{cases}
$$


Here, expression of (9) shows that the Heaviside function is the continuous function. If we take $\eta=0$, then the value of $\widetilde{\tilde{\rho}}_{i}(9)$ is degenerated to $\tilde{\rho}_{i}$ filter density which makes sure to minimize length scale on the solid phase that can be noted down as

$$
\rho_{i}=\left(1-e^{-\tilde{\beta \rho_{i}}}+\widetilde{\rho}_{i} e^{-\beta}\right)
$$

When $\eta=1$, then the behavior of projected physical density $\widetilde{\widetilde{\rho}}_{i}$ correspond to the modified Heaviside filter and performs right opposite to (10). It makes sure to minimize length scale on the void phase that can be written as

$$
\rho_{i}=\left(e^{-\beta\left(1-\tilde{\rho}_{i}\right)}-\left(1-\tilde{\rho}_{i}\right) e^{-\beta}\right) .
$$

Here, we describe concise and naiver manifestation that rests on the sigmoid function in spite of traditionally described using tanh function. The tanh function expression has already been defined $[29,30]$. The tanh-based function is described as

$$
\rho_{i}=\frac{\tanh (\beta \eta)+\tanh \left(\beta\left(\widetilde{\rho}_{i}-\eta\right)\right)}{\tanh (\beta \eta)+\tanh (\beta(1-\eta))} .
$$

The alternative expression provides easier and faster projection method of computing rather than the described threshold projection obtained from (9). This easier and shorter alternative sigmoid-based function expression can be written down as

$$
\begin{aligned}
\rho_{i} & =\frac{\sigma(\beta \eta)+\sigma\left(\beta\left(\widetilde{\rho}_{i}-\eta\right)\right)}{\sigma(\beta \eta)+\sigma(\beta(1-\eta))}, \\
\sigma(x) & =\frac{b}{1+e^{(-a \cdot *(x-c))}+d,} \\
\frac{\partial \rho_{i}}{\partial \widetilde{\rho}_{i}} & =\frac{(a \beta) \cdot * \sigma \beta\left(\widetilde{\rho}_{i}-\eta\right) \cdot *\left(1-\left(\sigma\left(\beta\left(\widetilde{\rho}_{i}-\eta\right)\right) / b\right)\right)-3 d}{\sigma(\beta \eta+\sigma(\beta(1-\eta))} .
\end{aligned}
$$

When $\beta$ approaches to $\infty$, then sigmoid-based function expression provides results similar to tanh-based function expression as obtained by (9). Here $a, b, c$, and $d$ are constant parameters. The comparison graph shows the behavior of sigmoid and tanh functions and is shown in Figure 1. In applied optimization procedure, parameter beta commands the sharpness of the projection in the transition zone. In order to make sure there is differentiability in the optimization, in addition, to avoid local minima, we construct a convenient proposal where the beta is gradually increased from 2 to 64 . Beta should be greater than zero; generally, parameter beta minimum value is 0.1 at first. Typically, the starting point value of parameter is $\beta=2$ which gradually increase two times $2 * \beta$ with respect to proceeding after each 50 number of iterations and the maximum value for $\beta$ is reached up to 64 . Figure 2 explains the behavior of $\widetilde{\tilde{\rho}}_{i}$ which depends on the threshold projection, parameters $\beta$ and $\eta$, respectively, and the initial design for MMB example, which slightly proceeds with respect to the parameter $\beta$ value.

Sigmund introduced a gray level indicator to measure the discreteness of the obtained optimized design [31]:

$$
M_{n d}=\frac{\sum_{i=1}^{n} 4 \rho_{i}\left(1-\rho_{i}\right)}{n} \times 100 \%
$$

If all elements' density distribution is equal to zero or one, the value of $M_{n d}=0 \%$; then, converged design outcomes will be completely discrete. On the contrary, if all element density distribution is equal to $0.5, M_{n d}=100 \%$; then, obviously converged design outcomes would be gray. However, the value of indicator $M_{n d}$ also differentiates in multiple projection phase cases. The obtained projection structure layout from equations (9) and (13) are basically impossible to differentiate with a little higher gray level for sigmoid projection at. So, we conclude that the $0 / 1$ threshold projections could not deliver suitable results in favor of complaint mechanism illustration.

\section{Robust Topology Optimization Formulation}

In topology optimization using the density-based method, the material distribution allocation is with respect to the density distribution vector $\widetilde{\tilde{\rho}}$. It is an idea already described by sigmoid 2009, i.e, to obtaining the robust topology optimization with regard to uniform dilated density. The dilated density is represented as $\widetilde{\widetilde{\rho}}^{d}=\widetilde{\widetilde{\rho}}^{d}(\rho)$, intermediate density $\widetilde{\widetilde{\rho}}^{i}=\widetilde{\widetilde{\rho}}^{i}(\rho)$, and eroded density $\widetilde{\widetilde{\rho}}^{e}=\widetilde{\widetilde{\rho}}^{e}(\rho)$. These densities can be expressed by applying the threshold projection with $1-\eta, 0.5$, and $\bar{\eta}$, respectively. The given optimization problem (1) can be written down as

$$
\begin{aligned}
\min _{\rho}: & \max f\left(\rho^{e}(\rho)\right), f\left(\rho^{i}(\rho)\right), f\left(\rho^{d}(\rho)\right), \\
: & K\left(\rho^{e}\right) u^{e}=f \\
: & K\left(\rho^{i}\right) u^{i}=f \\
: & K\left(\rho^{d}\right) u^{d}=f \\
& : f_{v}(\rho)=\frac{\sum_{i \in N_{e}} \rho_{i} v_{i}}{V} \leq V^{*} \\
& : 0 \leq \rho_{i} \leq 1 .
\end{aligned}
$$

The solution for three finite element problems needs to be acquired as the sensitivities of objective w.r.t. variables are 

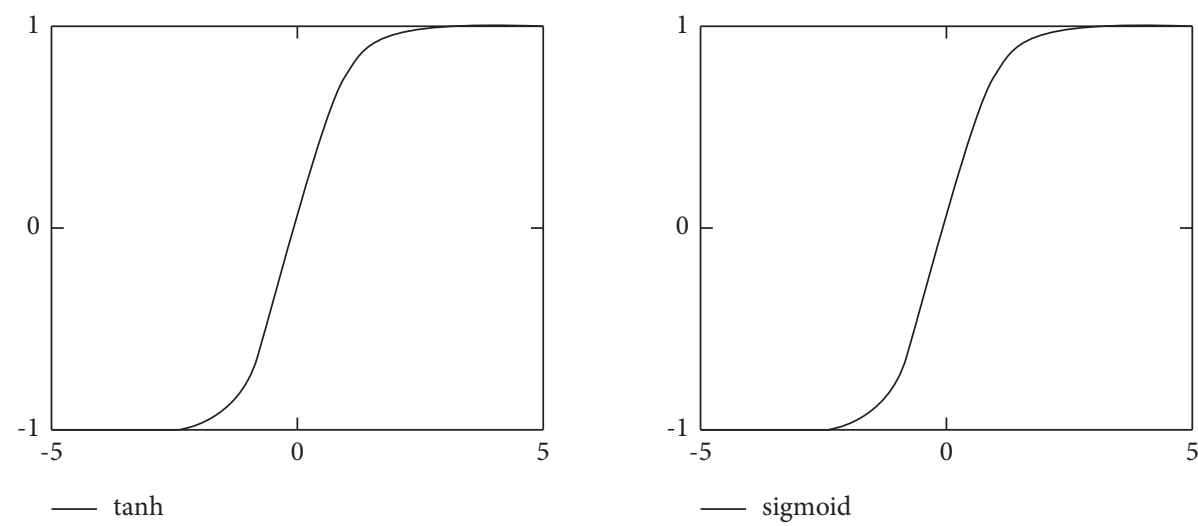

Figure 1: Activation function comparison graph.

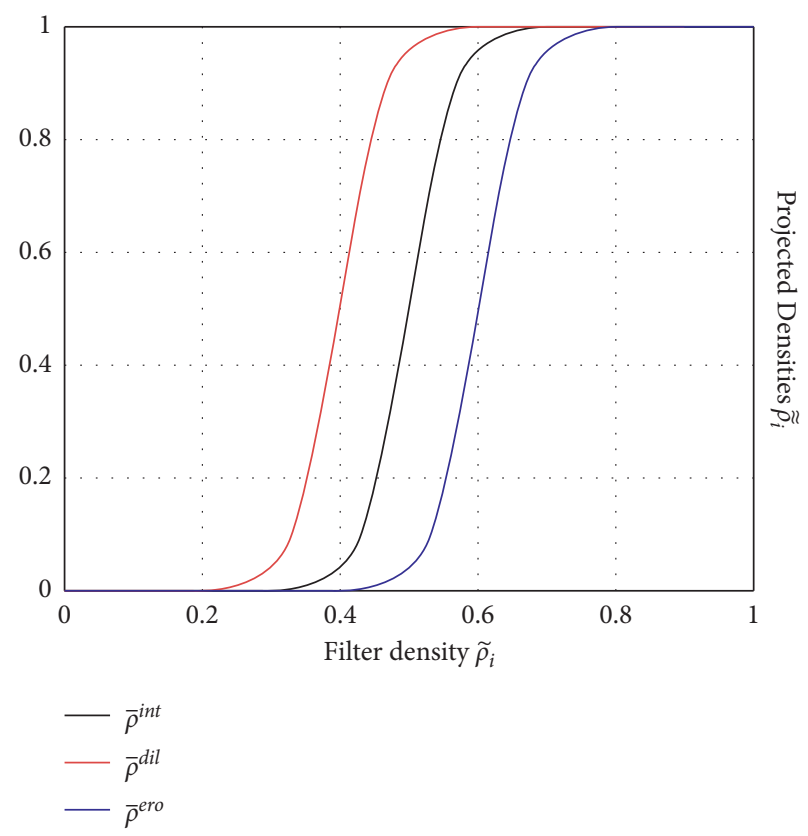

Figure 2: Heaviside filter effect.

dependent on the solution and utilization of the chain rule for the implementation of robust function. Figure 3 shows the impression of the three optimized design projections for efficient linear evolution density from 0 to 1 , respectively. The decrease in element size is obvious from the dilated case to the eroded case for a solid phase when the topology used for optimized designs is the same. At the same time the void phase tends to increase. To ensure the fulfilment of $\mathrm{min} / \mathrm{max}$ of the objective function, the manufacturing error bound for both phases are defined via difference between three designs.

\section{Numerical Analysis for Proposed Model}

4.1. MBB Cantilever-Density Filtering. In this section, there are numerous explanatory numerical examples to elaborate the worthy value of the suggested technique. As a usual go through for analysis, the execution of numerical solution methodology, the participating material properties, geometry data, and applied external loads are all given as dimensionless. Poisson's ratio and Young's modulus of the material are fixed as 0.3 and 1, respectively. To numerically solve the optimization of two-dimensional plane stress problems, we used the method of moving asymptotes as an optimizer. Figure 4 shows the initial structure domain of the MBB beam.

4.2. Density Filtering with Dimension $2 \times 1$. In this section, $2 \mathrm{D}$ cantilever beam examples are shown in the Figure 5 which demonstrates the feasibility of the proposed robust optimized topologies invention is useful. The design domain is represented with respect to different discretization nelx and nely and the number of elements in the horizontal and vertical direction; volume fraction is taken as 0.5 for all results. The particular penalization vector $P=3$ in addition to Young's modulus is 1 . The passion ration value is given as 0.3. This figure shows three density distributions eroded, intermediate, and dilated result. Results shown in Figure 5 


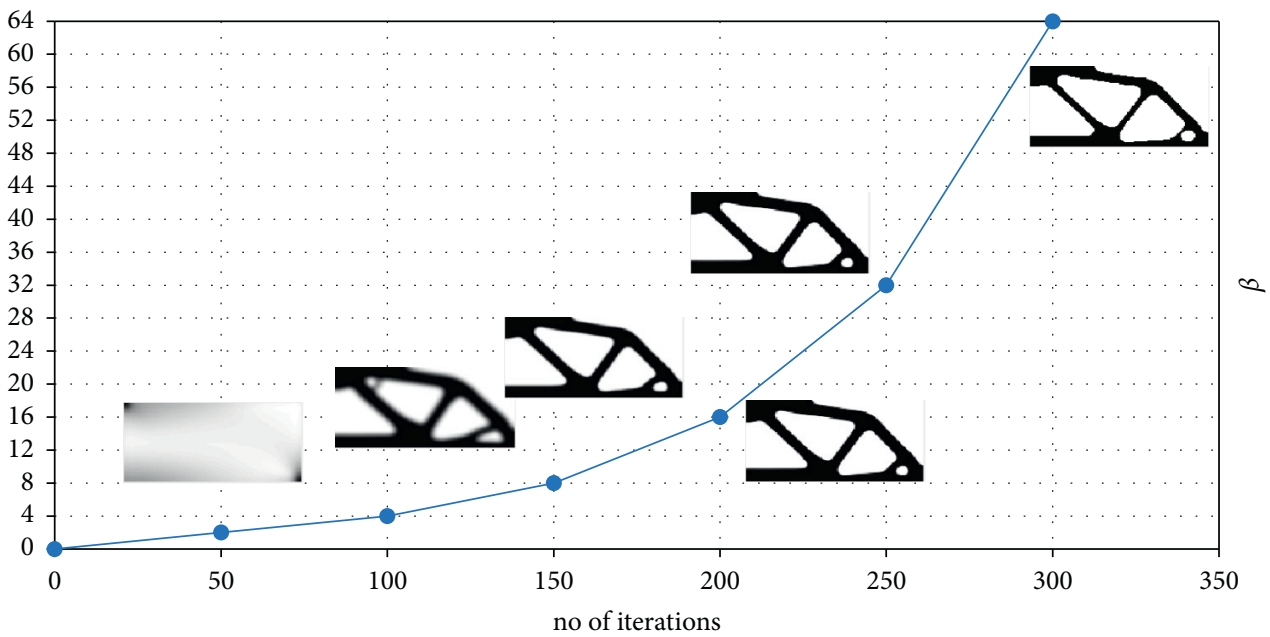

Figure 3: The initial design for MBB example.
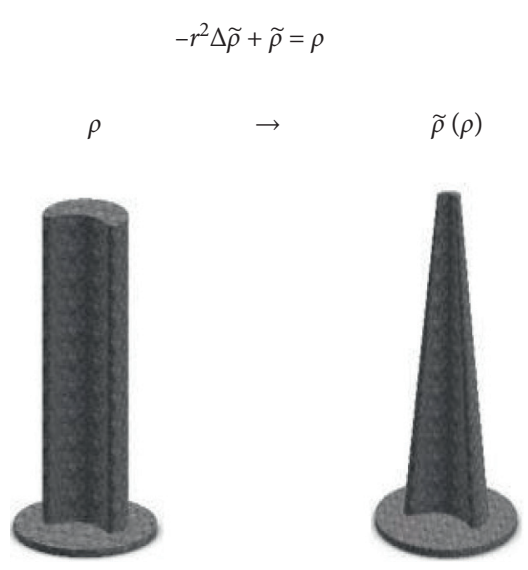

$$
\tilde{\rho}=\frac{\sigma(\beta \eta)+\sigma\left(\beta\left(\tilde{\rho}_{\mathrm{i}}-\eta\right)\right)}{\sigma(\beta \eta)+\sigma(\beta(1-\eta))} \mid
$$

Filtering

Projection

FIgURE 4: Robust topology optimization density filtering mesh cells for the beam with dimension $2 \times 1$.

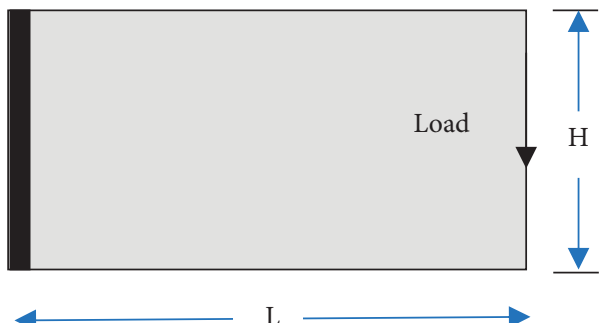

FIgURE 5: The initial structure domain of the MBB Beam.

depicts the robust, optimized formulation to make sure mesh-independent designs. The said designs and the morphology filters consist of matching properties such as that in conventional density filters carried out.

Figures 6(a) to 6(c) show that robust topology optimization has the same density filtering size, but mesh refinement cells go up for the beam with dimension $2 \times 1$, and
Figure 6(c) shows a little bit complicated topology optimized structure due to reducing half $r$ min value with the FE mesh cell. Figure 6 shows fine resolution result.

4.3. Density Filtering with Dimension $3 \times 1$. The initial optimized structure is shown in Figure 6. This figure also shows 
(i) nelx $=200$, nely $=100$

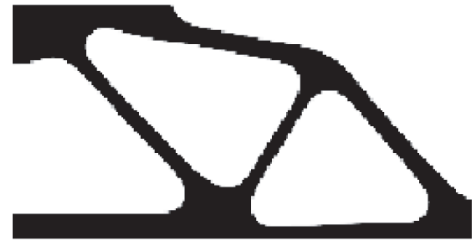

(a) Eroded design

$f=141.151$, volfrac $=0.5, \eta=0.25$

(ii) nelx $=400$, nely $=200$

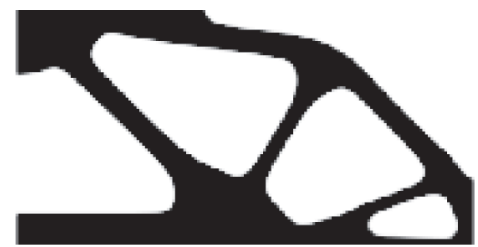

(d) Eroded design

$f=112.385$, volfrac $=0.5, \eta=0.25$

(iii) nelx $=400$, nely $=200$

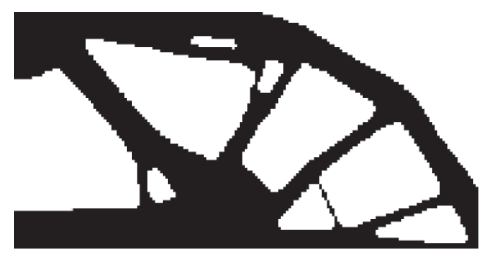

(g) Eroded design

$f=91.778$, volfrac $=0.5, \eta=0.25$

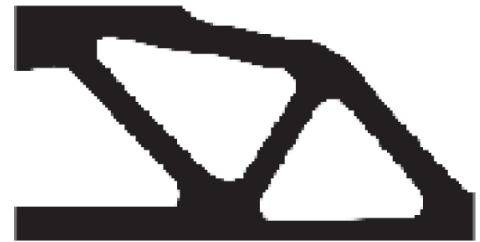

(b) Intermediate design

$f=101.985$, volfrac $=0.5, \eta=0.50$

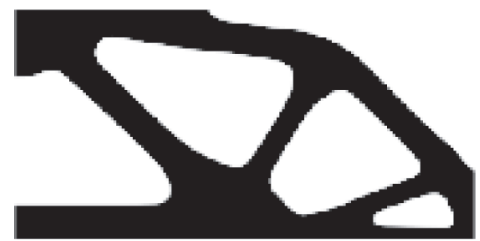

(e) Intermediate design $f=95.413$, volfrac $=0.5, \eta=0.5$

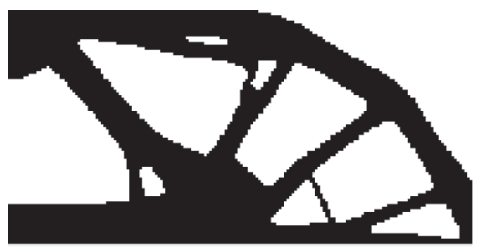

(h) Intermediate design $f=87.204$, volfrac $=0.5, \eta=0.5$

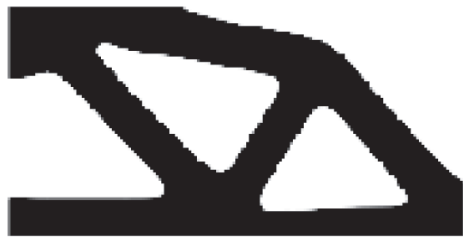

(c) Dilated design

$f=85.171$, volfrac $=0.5, \eta=0.75$

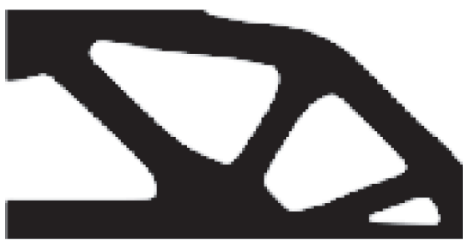

(f) Dilated design $f=85.684$, volfrac $=0.5, \eta=0.75$

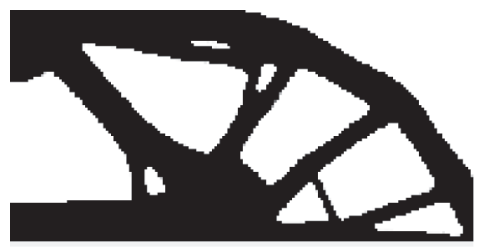

(i) Dilated design $f=84.134$, volfrac $=0.5, \eta=0$

FIGURe 6: Robust topology optimization density filtering mesh cells for the beam with dimension $2 \times 1$. (a)nelx $=200$, nely $=100$, (1) eroded design: $f=141.151$, volfrac $=0.5$, and $\eta=0.25$; (2) intermediate design: $f=101.985$, volfrac $=0.5$, and $\eta=0.50$; (3) dilated design: $f=85.171$, volfrac $=0.5$, and $\eta=0.75$; (b)nelx $=400$, nely $=200$, (1) eroded design: $f=112.385$, volfrac $=0.5$, and $\eta=0.25$; (2) intermediate design: $f=95.413$, volfrac $=0.5$, and $\eta=0.5$; (3) dilated design: $f=85.684$, volfrac $=0.5$, and $\eta=0.75$; (c)nelx $=400$, nely $=200$, (1) eroded design: $f=91.778$, volfrac $=0.5$, and $\eta=0.25$; (2) intermediate design: $f=87.204$, volfrac $=0.5$, and $\eta=0.5$; (3) dilated design: $f=84.134$, volfrac $=0.5$, and $\eta=0$.

three density distributions: eroded, intermediate, and dilated designs, and for the finite element design, domain is discretized as $300 \times 100,450 \times 150$, and $600 \times 200$ with dimension $4 \times 1$, respectively. Figures $7(\mathrm{a})-7(\mathrm{c})$ show that robust topology optimization has the same density filtering size, but mesh refinement cells go up for the beam with dimension $3 \times 1$, and Figure $7(\mathrm{~d})$ shows a little bit complicated topology optimized structure due to reducing half $r$ min with the FE mesh cell.

Here, $f$ denotes compliance. The compliance for eroded topology is always higher due to the repetitive dependence on volume fraction and material density in the objective function, and practically, the formulation of min-max for the compliance corresponds to minimizing, but there is no effect on the original and dilated one. Similarly, for the dilated topology, the compliance is low. For dilated, eroded, and intermediate, obtained designs have similar proposed topology for all cases. We suggested value $\eta=0.25$ for eroded, $\eta=0.5$ for intermediate, and $\eta=0.75$ for dilated design. This suggests that there is no vivid advantage of the robust design for a simple compliance minimizing problem. The obtained robust design is valuable for nano- and microfabrication. There is full control of the minimum solid and void length scale which makes a remarkable design by intermediate $\widetilde{\widetilde{\rho}}^{i}$ design. 
(i) nelx $=300$, nely $=100$

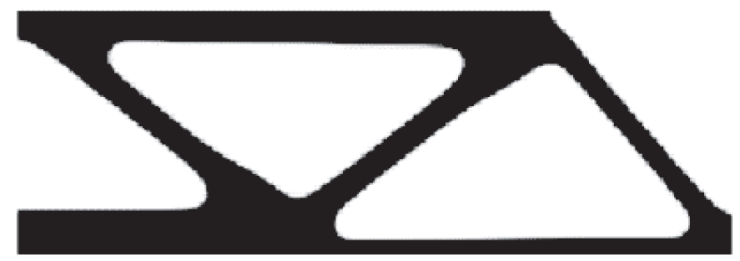

(a) Eroded design

$f=270.821$, volfrac $=0.5, \eta=0.25$

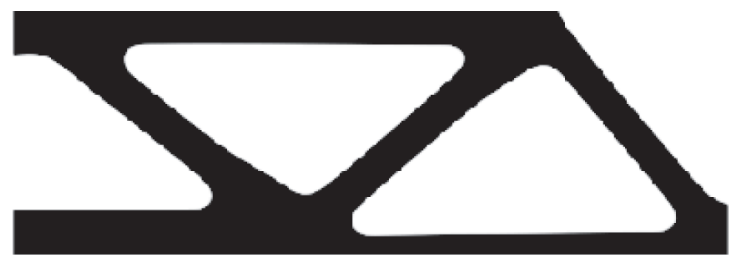

(b) Intermediate design

$f=221.427$, volfrac $=0.5, \eta=0.5$

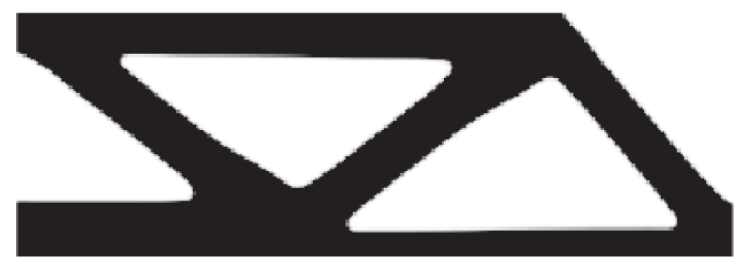

(c) Dilated design

$f=197.730$, volfrac $=0.5, \eta=0.75$

(iii) nel $x=600$, nely $=200$

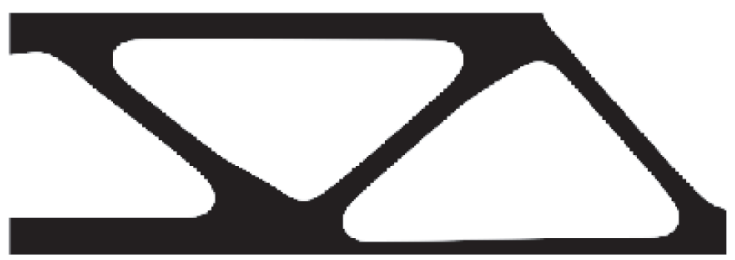

(a) Eroded design

$f=303.678$, volfrac $=0.5, \eta=0.25$

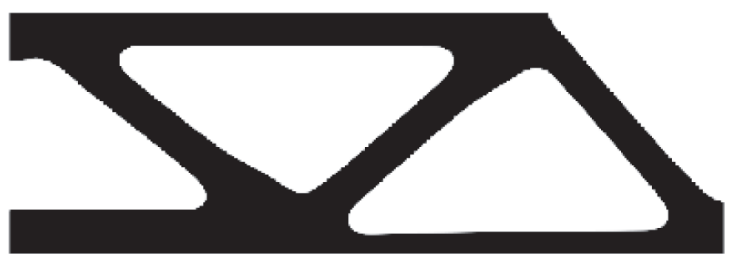

(b) Intermediate design

$f=239.584$, volfrac $=0.5, \eta=0.5$

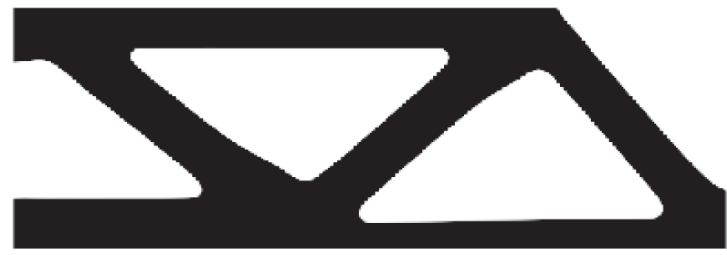

(c) Dilated design

$f=208.391$, volfrac $=0.5, \eta=0.75$ (ii) $n e l x=450$, nely $=150$

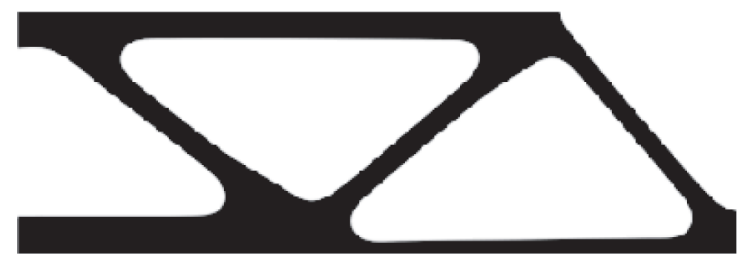

(a) Eroded design

$f=290.009$, volfrac $=0.5, \eta=0.25$

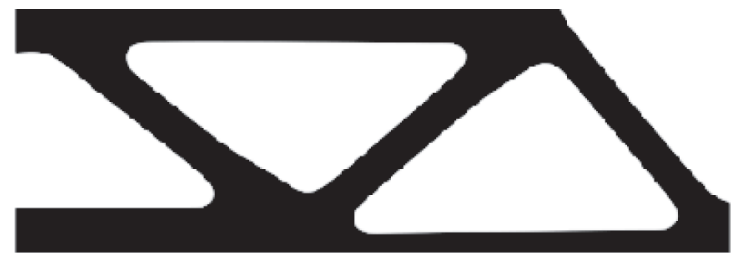

(b) Intermediate design

$f=231.488$, volfrac $=0.5, \eta=0.5$

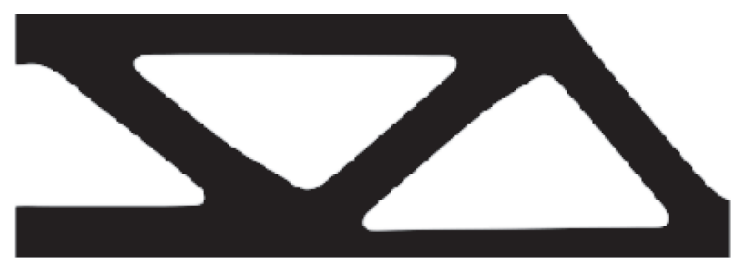

(c) Dilated design

$f=201.422$, volfrac $=0.5, \eta=0.75$

(iv) nel $=600$, nely $=200$

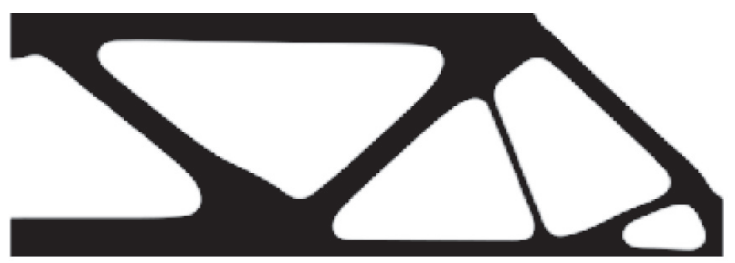

(a) Eroded design

$f=260.614$, volfrac $=0.5, \eta=0.25$

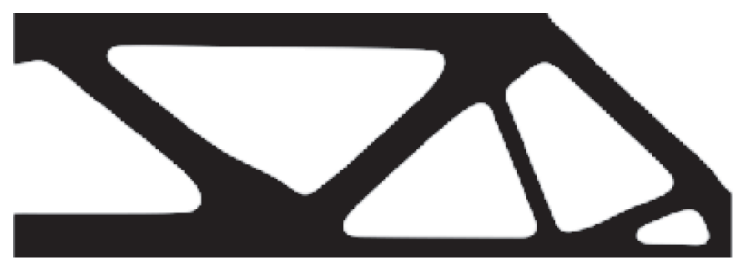

(b) Intermediate design

$f=225.917$, volfrac $=0.5, \eta=0.5$

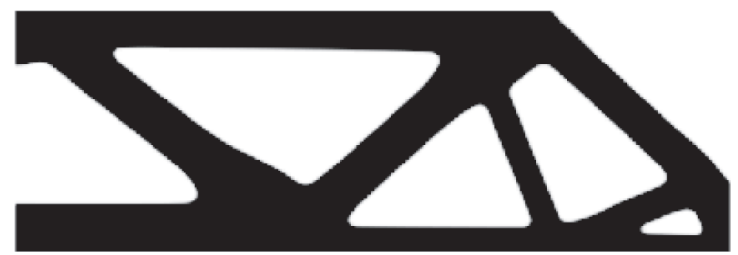

(c) Dilated design

$f=205.157$, volfrac $=0.5, \eta=0.75$

FIGURE 7: Robust topology optimization density filtering mesh cells for the beam with dimension $3 \times 1$. (a)nelx $=300$, nely $=100$; (1) eroded design: $f=270.821$, volfrac $=0.5$, and $\eta=0.25$; (2) intermediate design: $f=221.427$, volfrac $=0.5$, and $\eta=0.5$; (3) dilated design: $f=197.730$, volfrac $=0.5$, and $\eta=0.75$; (b)nelx $=450$, nely $=150$; (1) eroded design: $f=290.009$, volfrac $=0.5$, and $\eta=0.25$; (2) intermediate design: $f=231.488$, volfrac $=0.5$, and $\eta=0.5 ; \quad$ (3) dilated design: $f=201.422$, volfrac $=0.5$, and $\eta=0.75$; (c)nelx $=600$, nely $=200$; $\quad$ (1) $\quad$ eroded design: $f=303.678$, volfrac $=0.5$, and $\eta=0.25$; $\quad$ (2) intermediate design: $f=239.584$, volfrac $=0.5$, and $\eta=0.5$; (3) dilated design: $f=208.391$, volfrac $=0.5$, and $\eta=0.75 ;(\mathrm{d})$ nelx $=600$, nely $=200$; $(1)$ eroded design: $f=260.614$, volfrac $=0.5$, and $\eta=0.25$; (2) intermediate design: $f=225.917$, volfrac $=0.5$, and $\eta=0.5$; (3) dilated design: $f=205.157$, volfrac $=0.5$, and $\eta=0.75$. 


\section{Conclusion}

The suggested approaches, such as Heaviside projections and level set or phase-field methods, all proceed to smooth boundary representation and help to more clearly define topology results. The main purpose of this article is to introduce a new formulation to obtain minimum compliance in contrast to conventional scheme. The proposed method is much stable and all test models run with the same parameter settings. However, an added computational cost occurs to compute the three separate finite element problems. Designing more refined geometric representations and engineering models is suitable for subsequent shape and sizing. A transition from conceptual topology optimization to practical deployment of designs for emerging methods is required in the future. This can be done by employing engineering features in topology results, such as fillets, planes, and beam sections. A high-fidelity analysis in an automatic way would be extremely desirable in future for 3D structures.

\section{Data Availability}

The MATLAB source codes for all examples can be obtained from the corresponding author upon request.

\section{Conflicts of Interest}

The authors declare that they have no conflicts of interest to report with respect to the present study.

\section{Acknowledgments}

The authors are thankful to Professor Krister Svanberg for providing the program CODE of MMA. The authors wish to acknowledge the financial support from the National Natural Science Foundation of Beijing (2182067) and the Fundamental Research Funds of the Central Universities (2018ZD09).

\section{Supplementary Materials}

The MATLAB source codes for all examples are provided as per your contact to authors. (Supplementary Materials)

\section{References}

[1] M. P. Bendsoe and N. Kikuchi, "Generating optimal topologies in structural design using a homogenization method," Computer Methods in Applied Mechanics and Engineering, vol. 71, no. 2, pp. 197-224, 1988.

[2] J. D. Deaton and R. V. Grandhi, "A survey of structural and multidisciplinary continuum topology optimization: post 2000," Structural and Multidisciplinary Optimization, vol. 49, no. 1, pp. 1-38, 2014.

[3] A. Sohouli, A. Kefal, A. Abdelhamid, M. Yildiz, and A. Suleman, "Continuous density-based topology optimization of cracked structures using peridynamics," Structural and Multidisciplinary Optimization, vol. 62, pp. 2375-2389, 2020.

[4] W. Cheng, H. Wang, M. Zhang, and R. Du, "Improved proportional topology optimization algorithm for minimum volume problem with stress constraints," Engineering Computations, vol. 38, no. 1, pp. 392-412, 2020.

[5] B. S. Lazarov, F. Wang, and O. Sigmund, "Length scale and manufacturability in density-based topology optimization," Archive of Applied Mechanics, vol. 86, no. 1-2, pp. 189-218, 2016.

[6] K. Long, X. Wang, and H. Liu, "Stress-constrained topology optimization of continuum structures subjected to harmonic force excitation using sequential quadratic programming," Structural and Multidisciplinary Optimization, vol. 59, no. 5, pp. 1747-1759, 2019.

[7] S. Maruyama, S. Yamasaki, K. Yaji, and K. Fujita, “Topology optimization incorporating external variables with metamodeling," Structural and Multidisciplinary Optimization, vol. 62, pp. 1-12, 2020.

[8] W. Zhang, Y. Liu, Z. Du, Y. Zhu, and X. Guo, “A moving morphable component based topology optimization approach for rib-stiffened structures considering buckling constraints," Journal of Mechanical Design, vol. 140, no. 11, 2018.

[9] W. C. Parr, Introduction to Quality Engineering: Designing Quality into Products and Processes, Taylor \& Francis Group, Oxfordshire, UK, 1989.

[10] O. Bacarreza, M. H. Aliabadi, and A. Apicella, "Robust design and optimization of composite stiffened panels in postbuckling," Structural and Multidisciplinary Optimization, vol. 51, no. 2, pp. 409-422, 2015.

[11] C.-J. Thore, E. Holmberg, and A. Klarbring, "A general framework for robust topology optimization under loaduncertainty including stress constraints," Computer Methods in Applied Mechanics and Engineering, vol. 319, pp. 1-18, 2017.

[12] J. Guilleminot and C. Soize, "A stochastic model for elasticity tensors with uncertain material symmetries," International Journal of Solids and Structures, vol. 47, no. 22-23, pp. 3121-3130, 2010.

[13] G. C. Calafiore and F. Dabbene, "Optimization under uncertainty with applications to design of truss structures," Structural and Multidisciplinary Optimization, vol. 35, no. 3, pp. 189-200, 2008.

[14] J. Zhao and C. Wang, "Robust topology optimization under loading uncertainty based on linear elastic theory and orthogonal diagonalization of symmetric matrices," Computer Methods in Applied Mechanics and Engineering, vol. 273, pp. 204-218, 2014.

[15] K. Long, X. Wang, and Y. Du, "Robust topology optimization formulation including local failure and load uncertainty using sequential quadratic programming," International Journal of Mechanics and Materials in Design, vol. 15, no. 2, pp. 317-332, 2019.

[16] B. Xu, Y. Han, and L. Zhao, "Bi-directional evolutionary topology optimization of geometrically nonlinear continuum structures with stress constraints," Applied Mathematical Modelling, vol. 80, pp. 771-791, 2020.

[17] P. Geoffroy-Donders, G. Allaire, and O. Pantz, "3-D topology optimization of modulated and oriented periodic microstructures by the homogenization method," Journal of Computational Physics, vol. 401, Article ID 108994, 2020.

[18] Y. Gai, X. Zhu, Y. J. Zhang, W. Hou, and P. Hu, "Explicit isogeometric topology optimization based on moving morphable voids with closed B-spline boundary curves," Structural and Multidisciplinary Optimization, vol. 61, no. 3, pp. 963-982, 2020.

[19] N. Saeed, K. Long, and A. Rehman, "A review of structural optimization techniques for wind turbines," in Proceedings of the 2020 3rd International Conference on Computing, 
Mathematics and Engineering Technologies (iCoMET), pp. 18, Sukkur, Pakistan, 2020.

[20] Q. Xia and T. Shi, "Constraints of distance from boundary to skeleton: for the control of length scale in level set based structural topology optimization," Computer Methods in Applied Mechanics and Engineering, vol. 295, pp. 525-542, 2015.

[21] A. T. Gaynor and T. E. Johnson, "Eliminating occluded voids in additive manufacturing design via a projection-based topology optimization scheme," Additive Manufacturing, vol. 33, Article ID 101149, 2020.

[22] L. F. Qie, S. K. Jing, and R. C. Lian, "Heaviside-based morphological filters for topology optimization," In IOP Conference Series: Materials Science and Engineering, vol. 472, no. 1, Article ID 12034, 2019.

[23] L. Li and K. Khandelwal, "Volume preserving projection filters and continuation methods in topology optimization," Engineering Structures, vol. 85, pp. 144-161, 2015.

[24] O. Sigmund, "Manufacturing tolerant topology optimization," Acta Mechanica Sinica, vol. 25, no. 2, pp. 227-239, 2009.

[25] F. Wang, B. S. Lazarov, and O. Sigmund, "On projection methods, convergence and robust formulations in topology optimization," Structural and Multidisciplinary Optimization, vol. 43 , no. 6, pp. 767-784, 2011.

[26] T. E. Bruns and D. A. Tortorelli, "Topology optimization of non-linear elastic structures and compliant mechanisms," Computer Methods in Applied Mechanics and Engineering, vol. 190, no. 26-27, pp. 3443-3459, 2001

[27] B. Bourdin, "Filters in topology optimization," International Journal for Numerical Methods in Engineering, vol. 50, no. 9, pp. 2143-2158, 2001

[28] S. Xu, Y. Cai, and G. Cheng, "Volume preserving nonlinear density filter based on heaviside functions," Structural and Multidisciplinary Optimization, vol. 41, no. 4, pp. 495-505, 2010.

[29] N. Pollini and O. Amir, "Mixed projection- and density-based topology optimization with applications to structural assemblies," pp. 687-710, 2019, https://arxiv.org/abs/1906. 06512.

[30] T. E. Johnson and A. T. Gaynor, "Three-dimensional projection-based topology optimization for prescribed-angle selfsupporting additively manufactured structures," Additive Manufacturing, vol. 24, pp. 667-686, 2018.

[31] O. Sigmund, "Morphology-based black and white filters for topology optimization," Structural and Multidisciplinary Optimization, vol. 33, no. 4-5, pp. 401-424, 2007. 